\title{
Neue Wege der Beschreibung von Rechtstermini: der sema- siologisch-onomasiologische Ansatz der Begriffsbestimmung und seine Bedeutung für den Rechtsübersetzer
}

\author{
Eva Wiesmann \\ Universität Bologna/Forlì
}

Within the framework of modern terminological findings, a new approach for the description of legal terms is presented which attempts to overcome the limitations posed by the traditional conceptual understanding of Wüster's theory of terminology. The central question deals with the role of definition, which is of importance in the delimitation of legal terms, but more so and particularly in the formation of legal concepts. Viewed from the perspective of a modern conceptual understanding, legal concepts are the result, not the object of definition. Often, however, they may be formed through definitions only in the core area, but not in the marginal area important for the differentiation of legal concepts, and this means that further debate on the objects of law is required. By defining legal terms, and not legal concepts, a basic precondition is created for taking into consideration not only the subject specialist but also the linguistic context, and thus for fully taking into account the needs of the legal translator.

\section{Einleitung}

Im vorliegenden Beitrag, der zentrale Aspekte meiner Dissertation (Wiesmann 2004) aufgreift, wird vor dem Hintergrund moderner terminologiewissenschaftlicher Erkenntnisse ein neuer semasiologisch-onomasiologischer Ansatz der Bestimmung rechtlicher Begriffe vorgestellt, der neue Wege der Beschreibung von Rechtstermini eröffnet und für den Rechtsübersetzer mindestens in zweierlei Hinsicht von Bedeutung ist: Er erlaubt eine umfassende Begriffsbestimmung, dank derer rechtliche Begriffe nicht nur vollständig, sondern auch in all ihren Facetten und Dimensionen erfasst werden können. Bei diesem Ansatz können sowohl die fachlichen als auch die sprachlichen Kontexte Berücksichtigung finden, mit denen es der Rechtsübersetzer bei der Übersetzung von Rechtstexten zu tun hat. Der Beitrag nimmt seinen Ausgangspunkt in einem knappen Überblick über die Grundprinzipien der Allgemeinen Terminologielehre von Eugen Wüster, in dessen Mittelpunkt sein Begriffsverständnis und die damit verbundene Methode der Untersuchung steht. Im Anschluss daran wird dargelegt, welche Anpassungen bei der Anwendung der Wüsterschen Lehre auf die Rechtsterminologie von Peter Sandrini vorgenommen werden und auf welche Grenzen sein Ansatz stößt. Angesichts der auf den Punkt gebrachten Kritik an der traditionel- 
len Terminologielehre wird dann nach einem moderneren Begriffsverständnis gesucht, das für die Anwendung auf die Rechtsterminologie grundsätzlich geeigneter scheint, und es werden die erforderlichen Anpassungen der betreffenden terminologiewissenschaftlichen Erkenntnisse durchgeführt, die in den neuen Ansatz der Bestimmung rechtlicher Begriffe münden. Der Beitrag schließt mit zwei wesentlichen Konsequenzen, die sich daraus für die Verwaltung von Rechtstermini in einer Datenbank ergeben.

\section{Das Begriffsverständnis von Wüster}

Die Allgemeine Terminologielehre von Eugen Wüster, die in ihren Grundzügen in den 30er Jahren des 20. Jahrhunderts entstanden ist, hat die Auseinandersetzung mit den Begriffen besonders in der Naturwissenschaft und Technik maßgeblich beeinflusst. Sie war von mehr oder weniger großer Bedeutung für die verschiedenen terminologischen Schulen, die im vergangenen Jahrhundert entstanden sind und hat Eingang gefunden in von nationalen und internationalen Normungsinstituten erarbeitete terminologische Normen. Die Grundprinzipien der Wüsterschen Lehre lassen sich mit Temmerman (2000: 4-15) in den folgenden fünf Punkten zusammenfassen: 1) Terminologiearbeit beginnt nicht beim Terminus, sondern bei dem als sprachunabhängig gegeben betrachteten Begriff, was die onomasiologische Methode der Untersuchung bedingt, 2) die durch Abstraktion ausgehend von den Gegenständen der außersprachlichen Wirklichkeit gebildeten Begriffe haben einen geschlossenen Merkmalskomplex und sind daher in einem Begriffssystem genau gegeneinander abgrenzbar, 3) die Abgrenzung von Begriffen erfolgt entweder durch Inhalts-, durch Umfangs- oder durch Bestandsdefinitionen, dank derer die Position des Begriffs im Begriffssystem exakt angegeben werden kann, 4) Begriffe werden nach ihrer Bildung benannt, sind also vor den Benennungen gegeben, wobei die Beziehung zwischen Begriff und Benennung idealerweise eine eineindeutige, zumindest aber eine eindeutige ist, 5) für die Untersuchung von Begriffen ist nur die Synchronie relevant.

Was die Relationen zwischen den Gegenständen der außersprachlichen Wirklichkeit, den Begriffen und den sprachlichen Zeichen mit ihren Bedeutungen anbelangt, so werden sie von Wüster (1959/60: 188) in einem vierteiligen Modell erfasst. Entscheidend für ihn ist, dass die Beziehungen zwischen den Gegenständen und den Begriffen vorrangig, die Beziehungen zwischen den Bedeutungen und den Zeichen, die als Benennungen, aber auch in einer anderen semiotischen Form realisiert sein können, hingegen nachrangig sind, d.h. dem "sprachphilosophischlinguistischen Teil seiner Terminologielehre [ordnet Wüster] einen erkenntnistheoretischen Teil vor" (Oeser \& Budin 1999: 2173).

Während der für die begriffliche Abgegrenztheit Ausschlag gebende geschlossene Merkmalskomplex die Kontextautonomie eines solchen 
fachlichen Begriffs bedingt, heißt es in der traditionellen Terminologielehre vom nichtfachlichen Begriff, er werde erst durch den Zeichengebrauch, d.h. in einer Zeichensituation, aus der Bedeutung des Zeichens gebildet, entstehe also in jedem Kontext neu und anders und sei "ein Begriffskontinuum", das erst "in einer bestimmten Zeichensituation zum Begriff" (Felber 1995: 33) werde. Die beim nichtfachlichen Begriff getroffene Unterscheidung zwischen einer potentiellen und einer seinen Begriffsstatus determinierenden aktuellen Bedeutung wird beim fachlichen Begriff angesichts seiner postulierten Kontextautonomie aufgehoben. Der fachliche Begriff lässt sich, m.a.W., als festgelegte Bedeutung betrachten, die durch den Zeichengebrauch nicht beeinflusst wird. In der Inhaltsdefinition werden dabei die wesentlichen Merkmale des fachlichen Begriffs erfasst, in der Umfangsdefinition werden die Unterbegriffe eines Oberbegriffs aufgezählt und in der Bestandsdefinition sind die Gegenstände der außersprachlichen Wirklichkeit genannt, auf die sich der Begriff bezieht. Wegen der Vorrangigkeit des Begriffs vor dem als Benennung oder in einer anderen semiotischen Form realisierten Zeichen ist das Definiendum in allen drei Fällen der Begriff.

Eine solche Vorstellung von der Bildung, den Merkmalen und der Untersuchung des fachlichen Begriffs erklärt sich aus dem Ziel der sprachlichen Normung, das die von Wüster initiierte Terminologiearbeit verfolgt, bei der Synonymie und Polysemie zum Zweck der Verbesserung der Fachkommunikation ausgeschlossen werden und mit der in erster Linie den Fachleuten selbst und in zweiter Linie den Übersetzern gedient werden soll, die die Kommunikation zwischen Fachleuten über Sprachbarrieren hinweg ermöglichen.

\section{Sandrinis Anwendung der Allgemeinen Terminologielehre auf die Rechtsterminologie}

Der Versuch einer Anwendung der traditionellen Terminologielehre auf die Rechtsterminologie wurde erstmals von Peter Sandrini unternommen. Angesichts der Unterschiede, die zwischen den Terminologien der Naturwissenschaft und Technik und denen der Geistes- und Sozialwissenschaften bestehen und in Anbetracht der Besonderheiten, die die Rechtsterminologie auszeichnen, waren dabei eine Reihe von Anpassungen notwendig. Diesen Anpassungen liegt insbesondere die Feststellung der "Relativität der Rechtsbegriffe" (Sandrini 1996: 43) zugrunde, die sich aus der Institutionalität des immer an Rechtsordnungen gebundenen rechtlichen Handelns ergibt, das sich im Rahmen der Rechtsetzung, der Rechtsanwendung und der Rechtspraxis sprachlich unter Rückgriff auf Rechtstermini vollzieht und das Sprechen über Recht im Rahmen der Rechtslehre beeinflusst. Die insbesondere aus der Rechtsetzung hervorgehenden Rechtsvorschriften werden im Rahmen der Rechtsanwendung und der Rechtspraxis auf Lebenssachverhalte angewandt. Da sich diese immer 
wieder neu und anders stellen und nicht umfassend in den Rechtsvorschriften berücksichtigt werden können, geht jede Anwendung von Rechtsvorschriften mit deren Auslegung einher, für die eine gewisse Interpretationsoffenheit der Rechtssprache und mit ihr der Rechtstermini unabdingbar ist.

Für die rechtlichen Begriffe ergibt sich daraus zwangsläufig, dass sie strenggenommen nicht als durch Inhalts-, Umfangs- und Bestandsdefinitionen genau abgrenzbare fachliche Begriffe im Wüsterschen Sinne betrachtet werden können, was Sandrini zu folgenden Anpassungen hinsichtlich des Rechtsbegriffs und dessen Definition veranlasst: Er setzt rechtliche Begriffe mit den Tatbeständen von Normen gleich und hält fest, dass sie onomasiologisch ausgehend von den Lebenssachverhalten $\mathrm{zu}$ untersuchen und unter Einbeziehung des Normzwecks durch teleologische Begriffsdefinitionen zu bestimmen seien, die von ihm als Einheit von Tatbestand und Rechtsfolge angesehen werden.

Als eine teleologische Definition lässt sich zweifelsohne die Definition der Kündigung betrachten, die im Arbeitsrecht eine einseitige, empfangsbedürftige, rechtsgestaltende, bedingungsfeindliche, unwiderrufliche, formpflichtige Willenserklärung (Tatbestand) ist, durch die der Endtermin des Arbeitsverhältnisses (Rechtsfolge) bestimmt wird. Bereits bei der Definition des Arbeitnehmers als demjenigen, der - so der Tatbestand - aufgrund eines privatrechtlichen Vertrags oder eines ihm gleichgestellten Rechtsverhältnisses im Dienst eines anderen Arbeit leistet, zeigt sich jedoch, dass die mannigfaltigen Rechtsfolgen, die damit verbunden sind, weder in der vorliegenden, von Juristen erarbeiteten Definition erfasst sind noch in einer neuen Definition erfasst werden können, die im Rahmen der juristischen Terminologiearbeit erstellt werden sollte. Wird von den maßgeblichen gesetzgeberischen oder den mangels Legaldefinition erarbeiteten richterlichen und rechtswissenschaftlichen Definitionen ausgegangen, so muss, m.a.W., damit gerechnet werden, dass die Realität dem Ideal der teleologischen Definition nicht unbedingt gerecht wird. Soll das Ideal hingegen in einer neuen Definition Realität werden, so sind, wenn Definitionen - anders als bei der sog. enzyklopädischen Definition - auch strengen formalen Voraussetzungen genügen und insbesondere auf Definitionsgleichungen basieren sollen, u.U. schnell die Grenzen dessen erreicht, was sich überhaupt noch als Definition betrachten lässt.

Schaut man sich gute Rechtsenzyklopädien wie beispielsweise das Deutsche Rechts-Lexikon von Tilch \& Arloth (2001) an, die die vorliegenden gesetzgeberischen, richterlichen und rechtswissenschaftlichen Definitionen aufgreifen, so lässt sich in der Tat feststellen, dass Ausführungen zum Begriff vielfach von solchen zu den Rechtsfolgen getrennt werden. So heißt es dort beispielsweise zum Begriff der Anspruchskonkurrenz (Tilch \& Arloth 2001: 229): "Eine A. liegt dann vor, wenn ein Sachverhalt von mehreren anspruchsbegründenden Normen erfaßt wird, die dem Gläubiger alle einen auf dasselbe Ziel gerichteten 
Anspruch gewähren. [...].” $\mathrm{Zu}$ den damit verbundenen Rechtsfolgen wird hingegen ausgeführt: "In der Regel bestehen die Anspruchsgrundlagen unabhängig nebeneinander, dh jede ist ausschließlich nach ihren eigenen Voraussetzungen zu beurteilen; sind diese erfüllt, so entfällt der Anspruch nicht dadurch, daß außerdem ein weiterer auf dasselbe Ziel gerichteter Anspruch besteht. [...]."

Als problematisch muss darüber hinaus betrachtet werden, dass die Erfordernisse von Juristen und Rechtsübersetzern mit Blick auf die juristische Terminologiearbeit auseinanderfallen und sprachliche Normung nicht grundsätzlich, sondern nur in bestimmten Zusammenhängen, insbesondere in zwei- und mehrsprachigen Rechtsordnungen, anzustreben ist.

Vor allen Dingen im Rahmen des rechtlich-sprachlichen Handelns sind den Definitionen und der Definierbarkeit in verschiedener Hinsicht Grenzen gesetzt, die das Definiens und das Definiendum ebenso betreffen wie die Definitionsmacht der definierenden Juristen und den Zweck der von ihnen erarbeiteten Definitionen. Hinsichtlich des Definiens gibt es funktionsbedingt unbestimmte Rechtsbegriffe wie Treu und Glauben mit verhältnismäßig großen "Deutungs- und damit Bedeutungsspielräumen" (Busse 1999: 1383), die der Weiterentwicklung des Rechts und seiner Anpassung an die sich im Laufe der Zeit ändernden Gegebenheiten dienen, was eine Definition allenfalls bei der Rechtsanwendung, nicht aber bereits bei der Rechtsetzung sinnvoll macht. Was das Definiendum anbelangt, so ist es, wie sich unschwer am Beispiel Recht zeigen lässt, mitunter so komplex, dass eine umfassende Definition sich als ein schwieriges, wenn nicht gar unmögliches Unterfangen erweist oder mangels Legaldefinition nicht mit dem Konsens aller Juristen rechnen darf, wofür auch die Rede von der herrschenden Meinung und der herrschenden Lehre spricht. In Bezug auf die Definitionsmacht der definierenden Juristen setzt der Vorrang der gesetzgeberischen Definition den Rechtsanwendern und den Rechtswissenschaftlern insofern Grenzen, als sie an eine solche Definition grundsätzlich gebunden sind und sich nur in einem eng abgesteckten Rahmen davon entfernen dürfen. Die im Definitionszweck begründeten Grenzen schließlich hängen damit zusammen, dass Definitionen - wie im Fall von Sache in $\S 90$ des Bürgerlichen Gesetzbuchs (BGB) - vielfach nur in Bezug auf eine bestimmte Regelung oder aber auf einen bestimmten Einzelfall bezogen sind und daher keine allgemeine Gültigkeit für sich in Anspruch nehmen können.

Dass Definitionen im Recht und für Juristen nicht in Bezug auf alle rechtlichen Begriffe erforderlich sind, lässt sich auch wiederum in guten Rechtsenzyklopädien feststellen, wo die Ausführungen zum Begriff nicht unbedingt definitorischen Charakter haben. Als Beispiel sei hier der Begriff der Altersteilzeitarbeit angeführt, zu dem es in Tilch \& Arloth (2001: 147) heißt:

In Art 1 des Gesetzes zur Förderung des gleitenden Übergangs in den Ruhestand [...] war das Altersteilzeitgesetz [...] enthalten. Es ermöglicht 
Arbeitnehmern ab vollendetem 55. Lebensjahr die freiwillige Vereinbarung des Übergangs von einer Voll- zur Teilzeittätigkeit. Damit können sie ab dem 60. Lebensjahr vom Erwerbsleben in den Ruhestand gleiten. [...].

Auf völlig andere Voraussetzungen trifft dagegen eine juristische Terminologiearbeit, die den Rechtsübersetzer im Blick hat und die Sprachbarrieren zwischen Juristen überwinden will, die sich im Recht grundsätzlich als rechtssprachliche Barrieren gestalten. Eine solche Terminologiearbeit kommt um die Beschreibung von Rechtstermini nicht herum und erfordert Definitionen. Wie noch zu zeigen sein wird, sind diese Definitionen allerdings erstens sinnvollerweise keine Begriffs-, sondern über den Inhalt, den Umfang bzw. die Funktion Aufschluss gebende und fachliche wie sprachliche Kontexte berücksichtigende Nominaldefinitionen, haben also den Rechtsterminus selbst und nicht den rechtlichen Begriff als Definiendum. Zweitens wird der rechtliche Begriff bei strenger Berücksichtigung formaler Voraussetzungen, d.h. bei Zugrundelegung der Definitionsgleichung Definiendum = Definiens, durch sie u.U. nur im Kern- und nicht auch in dem für die Unterscheidung rechtlicher Begriffe wichtigen Randbereich bestimmt, was zum Zweck der umfassenden Begriffsbestimmung eine weiterführende, außerhalb des engen Rahmens der Definition stattfindende sprachliche Auseinandersetzung mit den Gegenständen des Rechts erforderlich macht. Drittens wird dem Rechtsübersetzer, wenn ihm durch die juristische Terminologiearbeit ein umfassender kognitiver Hintergrund für die in Angriff $\mathrm{zu}$ nehmende Übersetzung geliefert werden soll, am besten einerseits eine vollständige und eine rechtliche Begriffe in all ihren Facetten und Dimensionen erfassende Begriffsbestimmung präsentiert und andererseits wird ihm diese so präsentiert, dass daraus die Unterscheidung zwischen einer über den Kernbereich rechtlicher Begriffe Aufschluss gebenden Definition und einer die weiterführende Auseinandersetzung mit den Gegenständen des Rechts spiegelnden und den Randbereich rechtlicher Begriffe bestimmenden Vertiefung hervorgeht.

\section{Die Kritik an der Wüsterschen Terminologielehre}

Die Kritik an der Allgemeinen Terminologielehre Wüsters wird treffend in Temmerman (2000: 22) auf den Punkt gebracht. Ausgehend von den die fünf Grundprinzipien Wüsters in einigen oder allen Punkten in Frage stellenden terminologiewissenschaftlichen Erkenntnissen von Sager, Weissenhofer, Zawada und Swanepoel, Cabré, Meyer, Gaudin, Boulanger, Gambier und Kageura wird dort zudem die Notwendigkeit eines neuen Ansatzes einer soziokognitiven Terminologiewissenschaft begründet, der anschließend empirisch anhand der Untersuchung eines Korpus von biowissenschaftlichen Texten untermauert wird. Für den vorliegenden Beitrag kann aus den von Temmerman dargelegten und den von ihr 
gewonnenen Erkenntnissen mit Blick auf die Rechtsterminologie erstens festgehalten werden, dass das Begriffsverständnis der Wüsterschen Lehre und die den Begriffen zugeschriebene Vorrangigkeit vor den Benennungen dazu führt, dass nicht nur die Rolle der Benennungen in der Fachkommunikation, sondern auch deren Wichtigkeit für die menschliche Kognition verkannt wird, und zweitens, dass die daraus resultierende Idealvorstellung der kommunikativen und der kognitiven Realität insofern nicht standhalten kann, als eine nicht nur synchronische, sondern auch diachronische Untersuchung von Fachtexten als Produkten der Kommunikation zwischen Fachmann und Fachmann bzw. Fachmann und Nichtfachmann zu völlig anderen Ergebnissen führt, nämlich:

1) Termini haben in unterschiedlichen Textumgebungen unterschiedliche polyseme Bedeutungen (z.B. lassen sich dem Rechtsterminus Pacht die Bedeutungen 'Pachtvertrag', 'Pachtzeit' und 'Pachtzins' zuordnen), was die Wüstersche Unterscheidung zwischen fachlichem und nichtfachlichem Begriff relativiert;

2) Polysemie ist der Fachkommunikation eher zu- als abträglich, fördert sie doch das Verstehen von Begriffen in all ihren Facetten (wie bei der Pacht) und Dimensionen (wie beispielsweise beim Schuldverhältnis, worunter, so Musielak (1992: 74), sowohl das 'Recht auf Leistung' als auch das 'Rechtsverhältnis, aus dem sich die Forderungsbeziehungen zwischen den Beteiligten ergeben', zu verstehen ist, was verdeutlicht, dass die Forderungsbeziehungen Rechte auf Leistung implizieren: "Kraft des Schuldverhältnisses ist der Gläubiger", wie es in § 241 (1) BGB heißt, "berechtigt, von dem Schuldner eine Leistung zu fordern. Die Leistung kann auch in einem Unterlassen bestehen.”);

3) es gibt Begriffe (im Recht insbesondere die unbestimmten Rechtsbegriffe und die Typusbegriffe), die keinen geschlossenen Merkmalskomplex haben und die folglich auch nicht genau durch Definitionen, die ihren Inhalt mittels der Unterscheidung von den Begriffen wesentlichen Inhaltsmerkmalen fassen wollen, abgrenzbar sind (z.B. ist Treu und Glauben ein Grundsatz mit einer bestimmten Funktion, nämlich der, den in der Gemeinschaft herrschenden sozialethischen Wertvorstellungen Eingang ins Recht $\mathrm{zu}$ verschaffen, und dem Arbeitnehmer sind sowohl solche Merkmale zugeordnet, die als typisch angesehen werden, als auch solche, die Juristen ggf. als verzichtbar betrachten);

4) sowohl Begriffe als auch Benennungen laufen Entwicklungen durch, dabei bilden sich Begriffe in der und durch die Fachkommunikation, u.a. durch Definition, heraus und Benennungen spiegeln vielfach die Entwicklung, die sich dabei feststellen lässt (so ist der Begriff 'Einzelangaben über persönliche oder sachliche Verhältnisse einer bestimmten oder bestimmbaren natürlichen Person' heute an die Benennung personenbezogene Daten geknüpft, während früher, wie in Arntz et al. (2002: 128) herausgestellt wird, auch Benennungen wie z.B. personenbezogene Informationen, persönliche Daten oder private Daten 
gebräuchlich waren, die nicht unbedingt für genau den gleichen Begriff standen);

5) bei der Betrachtung eines bestimmten Status quo erlauben Synonyme dank je anders motivierter Benennungen eine unterschiedliche Sicht auf den betreffenden Begriff (die Benennungen nominelle Kapitalerhöhung und Kapitalerhöhung aus Gesellschaftsmitteln verdeutlichen im ersten Fall, dass es sich hierbei um einen Buchungsvorgang handelt, und im zweiten Fall, dass der Gesellschaft kein neues Kapital zufließt);

6) Metaphern fördern das Denken und damit die Kognition sowie das Verstehen im Rahmen der Kommunikation (Röhl zitiert in "Recht anschaulich'. Visualisierung in der Juristenausbildung" als Metaphern Beispiele wie Rechtsquelle, Schuldverhältnis als Bündel von Rechten, Richter als Subsumtionsautomat sowie die stark konventionalisierte Metapher Organ und die nur noch etymologisch als Metapher erklärbare Anfechtung von Willenserklärungen);

7) beim Verstehen von Begriffen ist von Unterschieden zwischen Fachmann und Nichtfachmann auszugehen (ein umfassendes Verstehen der Begriffe Bürgschaft und Garantievertrag und der Unterschiede, die zwischen ihnen bestehen, ist für Nichtfachleute aufgrund von Definitionen allein nicht möglich);

8) Eindeutigkeit und Eineindeutigkeit sind nicht nur angesichts der Wichtigkeit von Polysemie und Synonymie, sondern auch hinsichtlich der je unterschiedlichen Erfordernisse der naturwissenschaftlich-technischen Fächer auf der einen und der geistes- und sozialwissenschaftlichen Fächer auf der anderen Seite nicht grundsätzlich anstrebenswert (die Rede von der herrschenden Meinung und der herrschenden Lehre lässt u.a. erkennen, dass bei Juristen unterschiedliche Auffassungen über Begriffe bestehen können, die insofern Sinn machen, als damit zur Weiterentwicklung der Begriffe beigetragen wird).

Für Begriffe lässt sich auf dieser Grundlage mit Pozzi (1999: 3334) feststellen, dass es sich hierbei um sprachlich abgegrenzte, kulturbedingte menschliche Vorstellungen von der außersprachlichen Wirklichkeit handelt, die semasiologisch ausgehend von den Termini zu untersuchen und anhand von Nominaldefinitionen zu beschreiben sind, die nicht nur den fachlichen, sondern auch den sprachlichen Kontexten Rechnung tragen. Diese Erkenntnis ist auch für die Rechtsterminologie von grundsätzlicher Relevanz. Wie bereits angedeutet, sind diesbezüglich jedoch gewisse Anpassungen erforderlich.

\section{Der semasiologisch-onomasiologische Ansatz der Bestimmung rechtlicher Begriffe und seine Bedeutung für den Rechtsübersetzer}

In Anbetracht der Funktionsweise des Rechts, das Regelungen für die unterschiedlichsten Bereiche des menschlichen Lebens $\mathrm{zu}$ treffen hat und 
auf Lebenssachverhalte anzuwenden und dabei auszulegen ist, sind der Definition und der Definierbarkeit im Recht eine Reihe von Grenzen gesetzt, die einerseits dazu führen, dass in Bezug auf wichtige Rechtstermini keine Definition vorliegt, und andererseits dazu, dass es mehrere in unterschiedlichen Zusammenhängen oder von je anderen Juristen erarbeitete Definitionen geben kann, die sich auf andere rechtliche Begriffe oder auf verschiedene Facetten und Dimensionen ein und desselben Rechtsbegriffs beziehen.

Die besondere Rolle, die Definitionen im Recht und für Juristen spielen, zeigt sich dabei nicht erst bei der Bestimmung rechtlicher Begriffe, sondern bereits im Zusammenhang mit der Abgrenzung der Rechtsterminologie von der Gemeinsprache und von anderen Fachsprachen. Aus der zur Anwendung abstrakt formulierter Rechtsvorschriften auf konkrete Lebenssachverhalte erforderlichen Interpretationsoffenheit der Rechtssprache ergibt sich ihr besonders enges Verhältnis zur Gemeinsprache, während die Vielfalt der Regelungsbereiche, die von den Rechtsvorschriften betroffen sind, zu Überschneidungen nicht nur mit der Gemeinsprache, sondern auch mit anderen Fachsprachen führt. Legt man daher - wie das in der italienischen Rechtswissenschaft bei Scarpelli (1959) und Belvedere (1994) der Fall ist - bei der Abgrenzung der Rechtsterminologie sowohl die Regelungen einer Rechtsordnung als auch die in diesen berücksichtigten Regelungsbereiche zugrunde, so muss man zur Rechtsterminologie 1) Termini zählen, die wie legge (Gesetz) oder rilevante (erheblich) allein auf Rechtliches referieren, 2) Termini, die wie parentela (Verwandtschaft) oder gravidanza (Schwangerschaft) auf Tatsächliches und damit auf Außerrechtliches referieren, das der rechtlichen Regelung grundsätzlich zugänglich ist, und 3) Termini, die wie contratto (Vertrag) oder notaio (Notar) insofern auf Rechtliches und Tatsächliches gleichzeitig referieren, als sich von einem konkreten Sachverhalt bzw. einer konkreten Person erst dann sagen lässt, es handele sich hierbei um einen Vertrag bzw. einen Notar, nachdem festgestellt wurde, dass die rechtlichen Voraussetzungen hierfür erfüllt sind. Dass Definitionen folglich bei der Abgrenzung der Rechtsterminologie von der Gemeinsprache und von anderen Fachsprachen nicht das einzige Kriterium sein können, sondern die rechtssprachliche Bedeutung in aller Regel der gemeinsame Nenner ist, liegt auf der Hand: Während unverkennbar der Rechtssprache zugehörige Termini wie Treu und Glauben, gute Sitten oder Gemeinwohl einer juristischen Definition oder Termini wie Willenserklärung, Rechtsgeschäft oder Vertrag im BGB zumindest einer Legaldefinition entbehren, sind Termini wie Abfall, Emissionen oder Anlagen legaldefiniert, ihre Definition wurde, m.a.W., vom Gesetzgeber in dem und für das betreffende Gesetz erarbeitet. "Über eine schon vorliegende Fachsprachlichkeit legt sich also", wie von Soffritti (2002: 60) festgestellt wird, "die Fachsprachlichkeit, die den Norm setzenden juristischen Text kennzeichnet und in einem gewissen Sinne erst legitimiert." 
Für eine juristische Terminologiearbeit, die den Rechtsübersetzer im Blick hat, lässt sich daraus zweierlei ableiten: 1) juristische Definitionen sind eine wichtige, aber nicht einzige Grundlage, 2) mangels von Juristen erarbeiteter Definitionen sind die Definitionen im Rahmen der juristischen Terminologiearbeit zu erstellen.

Was spricht nun dafür, dass die im Rahmen der juristischen Terminologiearbeit zu erstellenden Definitionen keine Begriffs-, sondern entsprechend modernen terminologiewissenschaftlichen Erkenntnissen wie der von Pozzi Nominaldefinitionen sein sollten, als Definiendum also den Rechtsterminus mit seiner Bedeutung haben? Erstens, dass auch in Bezug auf die von Juristen erarbeiteten Definitionen festzustellen ist, dass sie grundlegend Nominaldefinitionen sind. Zweitens, dass eine semasiologische, vom Rechtsterminus ausgehende und nach dessen Bedeutung fragende Betrachtung neben der Berücksichtigung des fachlichen auch die des für den Rechtsübersetzer besonders wichtigen sprachlichen Kontextes ermöglicht. Und drittens schließlich, dass - wie bereits dargelegt wurde auch die angepasste Begriffsdefinition der Allgemeinen Terminologielehre im Recht auf bislang nicht überwundene Grenzen stößt.

Juristen unterscheiden, soweit zum ersten Punkt, i.d.R. zunächst zwischen der Nominal- und der mit der Begriffsdefinition in engem Zusammenhang stehenden Realdefinition und innerhalb der Nominaldefinition wird dann weiter zwischen der synthetischen oder Vereinbarungsdefinition als Festsetzung und der analytischen oder lexikalischen Definition als Feststellung differenziert, zwischen denen noch die Explikation als zum Zweck der Klärung erfolgende Festsetzung angesiedelt wird. Wegen ihres - mit dem Anspruch der Begriffsdefinition vergleichbaren - Anspruchs, das Wesentliche in der Definition fassen zu wollen, stößt die Realdefinition in Anbetracht der Komplexität der Gegenstände des Rechts bei Juristen eher auf Ablehnung. Dazu kommt, dass die im Recht maßgeblichen Definitionen des Gesetzgebers grundsätzlich synthetische Nominaldefinitionen sind, die normativ festsetzen, was der Gesetzgeber unter einem bestimmten Rechtsterminus in einem bestimmten gesetzlichen, für die Rechtsanwendung relevanten Zusammenhang verstanden wissen will, während die Definitionen, mit denen bei der Rechtsanwendung und in der Rechtswissenschaft die normativen, gesetzgeberischen Festsetzungen des Sprachgebrauchs oder mangels Legaldefinition der normative Sprachgebrauch des Gesetzgebers festgestellt wird, immer als analytische Nominaldefinitionen betrachtet werden müssen.

Rechtsübersetzer haben es, was den zweiten Punkt anbelangt, grundlegend mit Rechtstexten und folglich mit fachlichen und sprachlichen Kontexten zugleich zu tun. Wenn es nun der Realität solcher Texte entspricht, dass Rechtstermini in unterschiedlichen Textumgebungen unterschiedliche polyseme Bedeutungen haben können und dass polyseme Bedeutungen die Facetten und Dimensionen rechtlicher Begriffe spiegeln, dann muss es als sinnvoll erachtet werden, dass Rechtstermini mit ihren 
Bedeutungen definiert werden, die mangels bereits erarbeiteter juristischer Definitionen den fachlichen und zugleich sprachlichen Kontexten der Rechtstexte und damit dem juristischen Sprachgebrauch zu entnehmen sind.

Ein solcher semasiologischer Ansatz bedarf im Recht jedoch insofern einer onomasiologischen Ergänzung, als v.a. bei den auf Rechtliches und auf Tatsächliches gleichermaßen referierenden Rechtstermini durch Nominaldefinition nicht unbedingt eine vollständige Begriffsbestimmung möglich ist. Wenn beispielsweise auf der Grundlage von $\S 765$ (1) BGB Bürgschaft als 'Vertrag' definiert wird, 'durch den sich der Bürge gegenüber dem Gläubiger eines Dritten verpflichtet, für die Erfüllung der Verbindlichkeit des Dritten einzustehen', dann ist damit angesichts der Unmöglichkeit einer allein darauf basierenden genauen Abgrenzung von dem Begriff des Garantievertrags der Begriff der Bürgschaft nicht vollständig bestimmt, sondern es wird die Grundlage für die sprachliche Auseinandersetzung mit dem Gegenstand geschaffen, auf den sich der Rechtsterminus bezieht und mittels derer sich der damit verbundene rechtliche Begriff erst vollständig bestimmen lässt. $\mathrm{Zu}$ dieser weiterführenden Auseinandersetzung gehören der Inhalt der Bürgschaftspflicht, der Sicherungszweck der Bürgschaft, die Rechtsverhältnisse zwischen Gläubiger und Bürgen einerseits und zwischen Gläubiger und Hauptschuldner andererseits, die Wirksamkeitsvoraussetzungen und die Beendigung der Bürgschaft und die Formerfordernisse des Vertrags ebenso wie der akzessorische Charakter der Bürgschaft, d.h. die Abhängigkeit der Bürgenverpflichtung von der Hauptverbindlichkeit, die für die Abgrenzung der Begriffe Bürgschaft und Garantievertrag grundlegend ist. In einer der Definitionsgleichung Definiendum = Definiens entsprechenden Definition lässt sich diese Auseinandersetzung, wie unschwer zu erkennen ist, nicht mehr unterbringen.

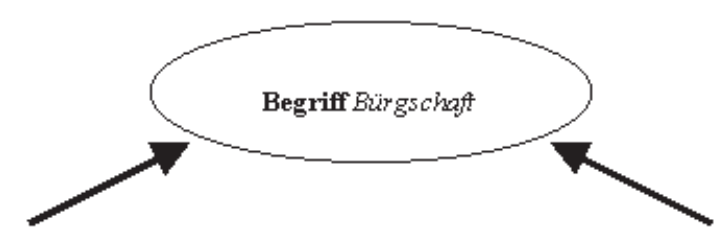

Definition des Terminus:

V ertrag, durch den sich der Bürge gegenüber dem Gläubiger eines Dritten verpflichtet, für die Erfüllung der V erbindlichkeit des Dritten einzustehen
Auseinandersetzung mit dem Gegenstand:

* Inhalt der Bürgschaftspflicht

> Sicherungszweck der Bürgschaft

* Rechtsverhältnisse zwischen Gläubiger und Bürgen und zwischen Gläubiger und $\mathrm{H}$ aupts chuldner

* akzessorischer Charakter der Bürgschaft

* usw.

ONOMASIOLOGISCHE PERSPEKTTVE

SEMASIOLOGISCHE PERS PEKTIVE

Abb. 1: Der Begriff der Bürgschaft 
Rechtliche Begriffe müssen daher als durch Nominaldefinitionen ggf. nur im Kernbereich entstehende und durch Nominaldefinitionen sprachlich nur im Kernbereich abgrenzbare juristische Vorstellungen von der außersprachlichen Wirklichkeit betrachtet werden, die hinsichtlich ihres Randbereichs u.U. erst aus der sprachlichen Auseinandersetzung der Juristen mit den Gegenständen des Rechts hervorgehen und erst auf dieser Grundlage vollständig gebildet und erfasst werden können. Wenn Rechtsübersetzer solche rechtlichen Begriffe, die sich durch Nominaldefinitionen nur im Kernbereich bestimmen lassen, umfassend verstehen sollen, dann ist zur umfassenden Beschreibung der betreffenden Rechtstermini auch die Information über die außerhalb des engen Rahmens der Definition stattfindende juristische Auseinandersetzung mit den Gegenständen des Rechts erforderlich, die zum kognitiven Hintergrund der Juristen, nicht hingegen der Rechtsübersetzer gehört.

Darüber hinaus impliziert die umfassende Beschreibung von Rechtstermini immer auch eine Untersuchung des juristischen Sprachgebrauchs, die mangels bereits vorliegender juristischer Definitionen die Grundlage für die im Rahmen der juristischen Terminologiearbeit zu erstellenden Definitionen ist. Diese führt erstens zu den rechtssprachlichen Bedeutungen, die, wie im Fall von Pacht, die verschiedenen Facetten des Begriffs spiegeln:

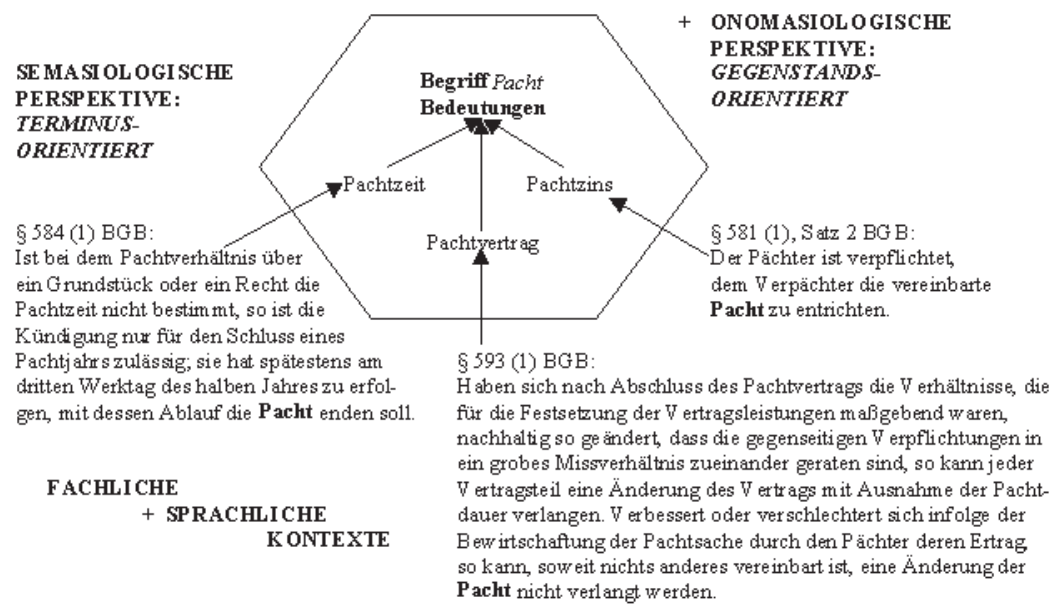

Abb. 2: Die Pacht - Begriff und Bedeutungen

Zweitens lässt sie, wie am Beispiel Schuldverhältnis verdeutlicht werden kann, die unterschiedlichen Dimensionen des Begriffs erkennen, die in einer engeren und einer weiteren Bedeutung zum Ausdruck kommen: 


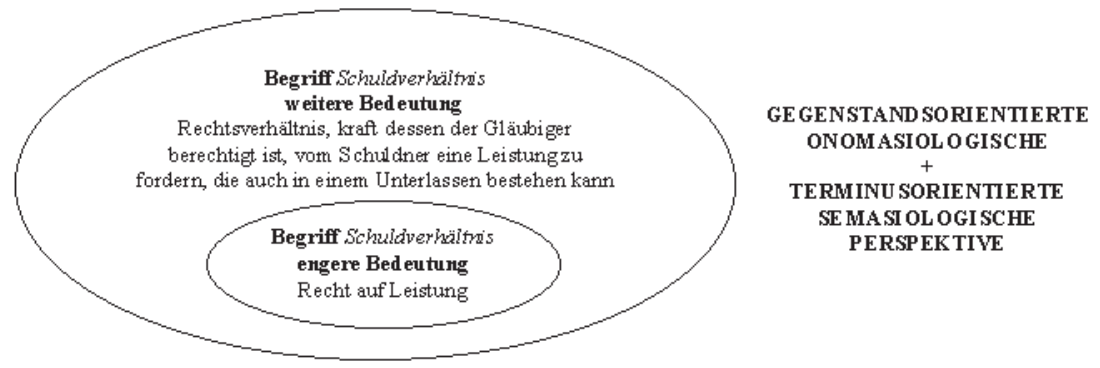

Abb. 3: Das Schuldverhältnis - engere und weitere Bedeutung

Und drittens schließlich kann auf diese Art und Weise auch den unterschiedlichen juristischen Definitionen Rechnung getragen werden, die sich mangels Konsens über den Begriff auf ein und denselben Rechtsterminus beziehen.

Nominaldefinitionen von Rechtstermini müssen dabei nicht nur über den Inhalt und den Umfang, sondern angesichts der unbestimmten Rechtsbegriffe wie Treu und Glauben auch über die Funktion Aufschluss geben, nämlich im Beispielfall der, den in der Gemeinschaft herrschenden sozialethischen Wertvorstellungen Eingang ins Recht zu verschaffen.

Ein umfassender kognitiver Hintergrund für die in Angriff zu nehmende Übersetzung wird dem Rechtsübersetzer nur durch die besagte umfassende Beschreibung von Rechtstermini geliefert, die sich auf fachliche wie auf sprachliche Kontexte stützt und damit seinen Bedürfnissen in vollem Umfang Rechnung trägt.

Für die im Anschluss an die juristische Terminologiearbeit in einer Datenbank zu erfassenden Daten und deren Präsentation ergibt sich daraus mindestens zweierlei:

1) im Vordergrund hat jeweils die Benennung zu stehen, der die verschiedenen Bedeutungen zugeordnet sind, die zum betreffenden Begriff gehören (z.B. Pacht: a - Pachtvertrag, b - Pachtzins, c - Pachtzeit),

2) die Definition eines Terminus ist von den Informationen zur Vertiefung zu trennen, die die weiterführende juristische Auseinandersetzung mit dem betreffenden Gegenstand spiegeln (z.B. Bürgschaft: a Vertrag, durch den sich der Bürge gegenüber dem Gläubiger eines Dritten verpflichtet, für die Erfüllung der Verbindlichkeit des Dritten einzustehen, b - Da der Pflicht des Bürgen keine Pflicht des Gläubigers gegenübersteht, ist der Bürgschaftsvertrag ein einseitig verpflichtender Vertrag, usw.).

Da es eine solche Datenpräsentation zudem erlaubt, die sich aus der Bindung der Rechtssprachen an Rechtsordnungen ergebenden Inkongruenzen zwischen den rechtlichen Begriffen unterschiedlicher Rechtsordnungen darzustellen, muss sie schließlich nicht nur für den Rechtsübersetzer, sondern auch für das Recht als geeignet erachtet werden. 


\section{Bibliographie}

Arntz, Reiner, Picht, Heribert \& Felix Mayer (2002). Einführung in die Terminologiearbeit. Studien zu Sprache und Technik. Hildesheim: Olms.

Belvedere, Andrea (1994). "Il linguaggio del codice civile: alcune osservazioni". Uberto Scarpelli \& Paolo Di Lucia (Hrsg.) (1994). Il linguaggio del diritto. Milano: LED, 403-452.

Busse, Dietrich (1999). "Die juristische Fachsprache als Institutionensprache am Beispiel von Gesetzen und ihrer Auslegung". Lothar Hoffmann, Hartwig Kalverkämper \& Herbert E. Wiegand (Hrsg.) (1999). Fachsprachen. Ein internationales Handbuch zur Fachsprachenforschung und Terminologiewissenschaft. 2. Halbbd. Handbücher zur Sprach- und Kommunikationswissenschaft. Berlin: de Gruyter, 1382-1391.

Felber, Helmut (1995). Allgemeine Terminologielehre und Wissenstechnik. Theoretische Grundlagen. IITF. Wien: Termnet.

Musielak, Hans-Joachim (1999). Grundkurs BGB. Eine Darstellung zur Vermittlung von Grundlagenwissen im bürgerlichen Recht mit Fällen und Fragen zur Lern- und Verständniskontrolle sowie mit Übungsklausuren. München: Beck.

Oeser, Erhard \& Gerhard Budin (1999). "Grundlagen der Terminologiewissenschaft". Lothar Hoffmann, Hartwig Kalverkämper \& Herbert E. Wiegand (Hrsg.) (1999). Fachsprachen. Ein internationales Handbuch zur Fachsprachenforschung und Terminologiewissenschaft. 2. Halbbd. Handbücher zur Sprach- und Kommunikationswissenschaft. Berlin: de Gruyter, 2171-2183.

Pozzi, María (1999). "The concept of 'concept' in terminology: a need for a new approach". Peter Sandrini (Hrsg.) (1999). Terminology and Knowledge Engineering. Proceedings of the Fifth International Congress on Terminology and Knowledge Engineering, 23-27 August 1999, Innsbruck, Austria. Vienna: TermNet, 28-42.

Röhl, Klaus F. “'Recht anschaulich'. Visualisierung in der Juristenausbildung”. Online unter:

http://www.ruhr-uni-bochum.de/rsozlog/Projekte/Visuelle\%20Rechtskommunikation/Inhaltsubersicht/Recht\%20anschaulich\%20Teil1\%20(Grundlagen).pdf (Zugriff am 10.08.2004).

Sandrini, Peter (1996). Terminologiearbeit im Recht. Deskriptiver begriffsorientierter Ansatz vom Standpunkt des Übersetzers. IITF. Wien: TermNet.

Scarpelli, Uberto (1959). Contributo alla semantica del linguaggio normativo. Torino: Accademia delle Scienze.

Soffritti, Marcello (2002). "Die doppelte Fachsprachlichkeit in aktuellen Norm setzenden Texten". Maurizio Gotti, Dorothee Heller \& Marina Dossena (Hrsg.) (2002). Conflict and Negotiation in Specialized Texts. Selected Papers of the $2^{\text {nd }}$ CERLIS conference. Linguistic Insights. Bern: Lang, 59-77.

Temmerman, Rita (2000). Towards New Ways of Terminology Description: The sociocognitive approach. Terminology and Lexicography Research and Practice. Amsterdam: John Benjamins.

Tilch, Horst \& Frank Arloth (Hgg.) (2001). Deutsches Rechts-Lexikon. Bd. 1: AF, Bd. 2: G-Q, Bd. 3: R-Z. München: Beck.

Wiesmann, Eva (2004). Rechtsübersetzung und Hilfsmittel zur Translation. Wissenschaftliche Grundlagen und computergestützte Umsetzung eines 
lexikographischen Konzepts. Forum für Fachsprachen-Forschung. Tübingen: Narr.

Wüster, Eugen (1959/1960). "Das Worten der Welt, schaubildlich und terminologisch dargestellt". Sprachforum 3-4, 183-204. 
